\title{
Vibrational Frequencies of Cerium Oxide-Bound CO: A Challenge for Conventional DFT Methods
}

\author{
Pablo G. Lustemberg, ${ }^{1,2}$ Philipp Pleßow, ${ }^{3}$ Yuemin Wang, ${ }^{4}$ Chengwu Yang, ${ }^{4}$ Alexei Nefedov, ${ }^{4}$ \\ Felix Studt, ${ }^{3}$ Christof Wöll, ${ }^{4, *}$ M. Verónica Ganduglia-Pirovano ${ }^{2, *}$ \\ ${ }^{1}$ Institute of Physics Rosario, IFIR, National Scientific and Technical Research Council, \\ CONICET, and National University of Rosario, UNR, S2000EKF Rosario, Santa Fe, Argentina \\ ${ }^{2}$ Institute of Catalysis and Petrochemistry, ICP, Spanish National Research Council, CSIC, \\ 28049 Madrid, Spain \\ ${ }^{3}$ Institute of Catalysis Research and Technology, IKFT, Karlsruhe Institute of Technology, KIT, \\ 76344 Eggenstein-Leopoldshafen, Germany
}

${ }^{4}$ Institute of Functional Interfaces, IFG, Karlsruhe Institute of Technology, KIT, 76344

Eggenstein-Leopoldshafen, Germany

\begin{abstract}
In ceria-based catalysis, the shape of the catalyst particle, which determines the exposed crystal facets, profoundly affects its reactivity. The vibrational frequency of adsorbed carbon monoxide $(\mathrm{CO})$ can be used as a sensitive probe to identify the exposed surface facets, provided reference data on well-defined single crystal surfaces together with a definitive theoretical assignment exist. We investigate the adsorption of $\mathrm{CO}$ on the $\mathrm{CeO}_{2}(110$ and (111) surfaces and show that the commonly applied $\mathrm{DFT}(\mathrm{PBE})+U$ method does not provide reliable $\mathrm{CO}$ vibrational frequencies by comparing with state-of-the-art infrared spectroscopy experiments for monocrystalline $\mathrm{CeO}_{2}$ surfaces. Good agreement requires the hybrid DFT approach with the HSE06 functional. The failure of conventional DFT is explained in terms of its inability to accurately describe the facet- and configuration-specific donation and backdonation effects that control the changes in the $\mathrm{C}-\mathrm{O}$ bond length upon $\mathrm{CO}$ adsorption and the $\mathrm{CO}$ force constant. Our findings thus provide a theoretical basis for the detailed interpretation of experiments and open up the path to characterize more complex scenarios, including oxygen vacancies and metal adatoms.
\end{abstract}

${ }^{*}$ Corresponding author

christof.woell@kit.edu,vggp@icp.csic.es

Cerium dioxide $\left(\mathrm{CeO}_{2}\right.$ or ceria) is extremely important in catalysis, either as a catalyst or as a support material [1], as well as in many other applications such as oxide-ion conductors in solid-oxide fuel cells [2] and lately in biology [3,4]. It is known that the ceria surface structure may alter the catalytic activity of ceria-based catalysts [5], and thus, ceria nanocrystals with controlled morphologies, such as nanooctahedra ( $\{111\}$ facet), nanocubes ( $\{100\}$ facet), and nanorods ( $\{100\}$ and $\{110\}$ facets) are fabricated [6], and their chemisorption and reactivity properties investigated [7-12]. However, under reaction conditions ceria facets can undergo restructuring [13-17]. These findings make it crucial to develop characterization methods that are 
able to probe the surface of ceria-based catalysts with high specificity under operando conditions [18]. The characterization of oxide surfaces via a characteristic shift of the vibrational band of small probe molecules has high potential in this regard but crucially depends on highly accurate reference data.

$\mathrm{CO}$ is -in principle - a useful probe molecule and thus a fairly large number of infrared spectroscopy (IR) studies for ceria powder samples exposed to CO have been reported [19-23]. However, for a reliable assignment of $\mathrm{CO}$ vibrational frequencies, as required for example in the surface-ligand IR (SLIR) approach to characterize oxide particles [24], reference data for CO adsorbed on well-defined single crystal surfaces together with a definitive theoretical assignment are required. Unfortunately, theoretical methods, largely depending on density functional theory (DFT), have been lacking behind in the accurate description of the small shift of the $\mathrm{C}-\mathrm{O}$ stretching frequency induced by its weak interaction with the oxide. This has been the motivation for pursuing experimental and theoretical research on the adsorption of $\mathrm{CO}$ onto extended monocrystalline ceria surfaces [25-31]. As of yet, studies that combine computational modelling and experiment are limited to $\mathrm{CO}$ adsorption on the (111) surface $[25,26]$. For one monolayer (ML) $\mathrm{CO}$ adsorption on the oxidized $\mathrm{CeO}_{2}(111)$ surface, there is a blue shift $(\Delta \mathrm{v})$ of $+11 \mathrm{~cm}^{-1}$ with respect to the $\mathrm{CO}$ vibrational stretching frequency in the gas phase [25], a common scenario for $\mathrm{CO}$ adsorbed on metal oxides [32]. The latest reported theoretical $\Delta v$ value of $+9 \mathrm{~cm}^{-1}$ [26] is in good agreement with experiment; it has been obtained by employing dispersion-corrected density-functional theory (DFT) in the DFT(PBE) $+U(4.5)$ approach [33], with the PBE (PerdewBurke-Ernzerhof) functional [34] and an effective Hubbard $U$-like term of $4.5 \mathrm{eV}$ added for the $\mathrm{Ce} 4 f$ states [35]. However, this apparently good agreement is limited to a very diluted $\mathrm{CO}$ adlayer (1/16 ML), since for full coverage, the agreement is less satisfying, as we will show below.

The DFT(PBE) approach, which is based on the generalized gradient approximation (GGA) for the exchange-correlation energy, is known to badly fail in accurately describing reduced ceria [36-38], whereas $\mathrm{DFT}(\mathrm{PBE})+U$, is commonly applied in the study of ceria-based systems, but questions regarding the best value for the $U$ parameter are still under debate $[35,38$ 42]. However, there is consensus that hybrid functionals using mixtures of DFT(GGA) and Hartree-Fock exchange energies (e.g., the HSE06 functional [43]) yield higher computational accuracy for ceria-based systems [38, 42, 44, 45, 46, 47]. Whether hybrid DFT is able to resolve the problems in describing $\mathrm{CO}$ adsorption on differently oriented ceria surfaces described above, that is the question. The use of hybrid functionals has rarely been considered crucial for the description of oxide-bound $\mathrm{CO}$, with the exception of $\mathrm{CO}$ on $\mathrm{MgO}(001)[48,49]$, and we recall that hybrid DFT has helped resolve the $\mathrm{CO} / \mathrm{Pt}(111)$ puzzle associated with the failure of DFT(GGA) approaches to properly describe CO-metal interactions [50]. 
In this Letter, we present a detailed analysis of $\mathrm{CO}$ adsorbed on the $\mathrm{CeO}_{2}(111)$ and (110) surfaces and demonstrate that the widely used $\mathrm{DFT}(\mathrm{PBE})+U$ approach is not well suited for the prediction of $\mathrm{CO}$ vibrational frequencies. While being computationally fairly inexpensive, in some cases this method even is in qualitative disagreement with experiment, i.e., instead of the typical blue shift of $\mathrm{CO}$ on oxides, a red shift is predicted. We provide firm computational evidence that for $\mathrm{CO}$ adsorbed on ceria surfaces the use of hybrid functionals is mandatory. It is only at this level of theory that the experimentally observed larger blue shift for $\mathrm{CeO}_{2}(110)$ by 17 $\mathrm{cm}^{-1}$ [15] compared to $\mathrm{CeO}_{2}(111)$, is recovered. A careful analysis allows to relate the failure of the simpler $\mathrm{DFT}(\mathrm{PBE})+U$ approach to the inability of the PBE functional to provide an accurate description of details of the electronic structure, in particular the facet- and configuration-specific donation and backdonation effects that control the changes in the $\mathrm{C}-\mathrm{O}$ bond length upon $\mathrm{CO}$ adsorption and the $\mathrm{CO}$ force constant.

Moreover, the combination of high-resolution $p$-polarized IRRAS data with the results of DFT(HSE06) calculations allows us to gain unique insight into the $\mathrm{CO}$ adsorption modes on ceria surfaces. In Fig. 1 we show the results of IRRAS experiments of $1 \mathrm{ML}$ adsorbed $\mathrm{CO}$ on single crystalline $\mathrm{CeO}_{2}(111)$ and $\mathrm{CeO}_{2}(110)$ surfaces. For $\mathrm{CeO}_{2}(111)$ a single intense band is visible for $p$-polarization at $2154 \mathrm{~cm}^{-1}$ (Fig. 1, red circles), blue shifted $\Delta \mathrm{v}=+11 \mathrm{~cm}^{-1}$ relative to the gas phase $\left(2143 \mathrm{~cm}^{-1}\right.$ [51]). In previous work, this band has been assigned to $\mathrm{CO}$ bound to $\mathrm{Ce}^{4+}$ cations embedded in a perfect single-crystalline $\mathrm{CeO}_{2}(111)$ surface environment [25]. For the $\mathrm{CeO}_{2}(110)$ surface, the CO SLIR data (Fig. 1, blue circles), reveal a more complex situation. For the saturated surface, a strong negative band is seen for $p$-polarization at $2071 \mathrm{~cm}^{-1}$, accompanied by a smaller positive feature at $2160 \mathrm{~cm}^{-1}$. Clearly, the data for the (110) surface are not consistent with just one peak. In fact, a fit using a negative band at $2171 \mathrm{~cm}^{-1}$ and a positive band at $2160 \mathrm{~cm}^{-1}$ (Fig. 1, upper panel, black line) yields good agreement with the data. In earlier work [15], the higher frequency peak $\left(2171 \mathrm{~cm}^{-1}\right)$ has been tentatively assigned to $\mathrm{CO}$ bound to $\mathrm{Ce}^{4+}$ cations, while the weaker peak at $2160 \mathrm{~cm}^{-1}$, indicating the presence of a second species, has not been discussed. The sign of the feature (positive rather than negative) indicates the presence of a strongly tilted species with a substantial component of the transition dipole moment oriented parallel to the substrate.

Apart from $\mathrm{CO}$ on $\mathrm{MgO}(001)$ [49)], the presence of a strongly tilted $\mathrm{CO}$ species at a cationic site on a low indexed single crystal oxide surface is uncommon [52]. In addition, it is surprising that the vibrational frequency of $\mathrm{CO}$ bound to $\mathrm{Ce}^{4+}$ sites differs by almost $20 \mathrm{~cm}^{-1}$ between (111) and (110) surfaces - in many previous works it has been assumed that these frequencies are mainly sensitive to the charge state of the metal ion; see [24] and references therein. A thorough understanding of the binding of small reactants such as $\mathrm{CO}$ on oxide surfaces 
is of fundamental interest and essential if $\mathrm{CO}$ is used as an IR probe molecule for determination of exposed surface facets.

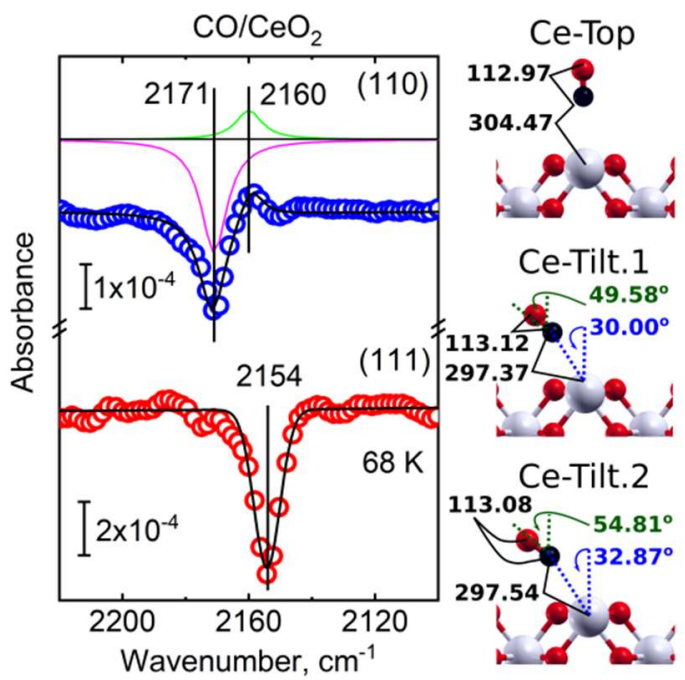

Figure 1. IRRAS spectra of $1 \mathrm{ML} \mathrm{CO}$ adsorption on oxidize $\mathrm{CeO}_{2}(111)$ (red circles) and $\mathrm{CeO}_{2}(110)$ (blue circles) surfaces with $p$-polarized light as well as HSE06 optimized $\mathrm{CO}-\mathrm{CeO}_{2}(110)$ structures. Selected interatomic distances (in pm) are indicated. Black lines show the corresponding fits. The upper panel presents two-peaks deconvolution, corresponding to bands at $2171 \mathrm{~cm}^{-1}$ (magenta) and $2160 \mathrm{~cm}^{-1}$ (green). Adapted with permission from [25], Copyright 2014 Royal Society of Chemistry and with permission from [15], Copyright 2017 John Wiley \& Sons, Inc.

We have first analyzed the binding of $1 \mathrm{ML} \mathrm{CO}$ on the $\mathrm{CeO}_{2}(110)$ and (111) surfaces by performing high-precision DFT(PBE) $+U(4.5)$ calculations (see Supporting Information). To our surprise, for the most strongly bound $\mathrm{CO}$ species on the $\mathrm{CeO}_{2}(110)$ surface, instead of the typical blue shift of $\mathrm{CO}$ adsorbed on metal oxides, the results revealed a distinct $\left(-23 \mathrm{~cm}^{-1}\right)$ red shift (Table 1), and for $\mathrm{CO}$ adsorbed on $\mathrm{CeO}_{2}(111)$, as already mentioned, the shift is about zero $(+1$ $\mathrm{cm}^{-1}$ ), in pronounced contrast to experimental observations (Fig. 1). At this point, we must come to the conclusion that theory at the $\mathrm{DFT}(\mathrm{PBE})+U$ level is not adequate to describe the bonding situation of $\mathrm{CO}$ on ceria surfaces. 
Table 1. Calculated DFT energies for the binding of $1 \mathrm{ML} \mathrm{CO}$ to the $\mathrm{CeO}_{2}(111)$ and (110) surfaces with $(1 \times 1)$ periodicity, scaled $\mathrm{CO}$ stretching vibrational frequency (by the method-dependent factor $\lambda=v_{C O_{\text {gas }}}^{\text {exp }} / v_{C O_{\text {gas }}}^{\text {calc }}$ with $v_{C 0_{\text {gas }}}^{\text {exp }}=2143 \mathrm{~cm}^{-1}$ [51] and $v_{C O_{\text {gas }}}^{\text {calc }}$ as given in Table S2), frequency shift, $\Delta \mathrm{v}$, and change in the $\mathrm{C}-\mathrm{O}$ bond length, $\Delta d_{\mathrm{C}-\mathrm{O}}$, upon adsorption with respect to the calculated values for the gas phase molecule, respectively. $d_{\mathrm{Ce}-\mathrm{C}}$ is the distance between the $\mathrm{C}$ and the $\mathrm{Ce}^{4+}$ site to which the $\mathrm{CO}$ is attached.

\begin{tabular}{|c|c|c|c|c|c|c|}
\hline Method & Site & $\begin{array}{r}\mathbf{E}_{a d s} \\
(\mathrm{eV})\end{array}$ & $\begin{array}{l}d_{\mathrm{Ce}-\mathrm{C}} \\
(\mathbf{p m})\end{array}$ & $\begin{array}{c}\Delta d_{\mathrm{C}-\mathrm{O}} \\
(\mathrm{pm})\end{array}$ & $\begin{array}{c}u \\
\left(\mathbf{c m}^{-1}\right)\end{array}$ & $\begin{array}{c}\Delta v \\
\left(\mathbf{c m}^{-1}\right)\end{array}$ \\
\hline \multicolumn{7}{|c|}{ (111) } \\
\hline IRRAS & & & & & 2154 & +11 \\
\hline $\mathrm{PBE}+U(4.5)$ & atop & -0.03 & 288 & +0.05 & 2144 & +1 \\
\hline HSE06 & atop & -0.09 & 295 & -0.06 & 2157 & +14 \\
\hline \multicolumn{7}{|c|}{ (110) } \\
\hline IRRAS & & & & & $2171 / 2160$ & $+28 /+17$ \\
\hline \multirow[t]{3}{*}{$\mathrm{PBE}+U(4.5)$} & atop & -0.16 & 299 & -0.12 & 2151 & +8 \\
\hline & tilt.1 & -0.22 & 290 & +0.09 & 2120 & -23 \\
\hline & tilt.2 & -0.21 & 291 & +0.06 & 2130 & -13 \\
\hline \multirow[t]{3}{*}{ HSE06 } & atop & -0.15 & 304 & -0.18 & 2164 & +21 \\
\hline & tilt.1 & -0.20 & 297 & -0.03 & 2145 & +2 \\
\hline & tilt.2 & -0.19 & 298 & -0.07 & 2150 & +7 \\
\hline
\end{tabular}

In order to resolve this issue, we have conducted a systematic study employing different levels of theory and in particular using hybrid functionals. We have first applied DFT with a number of semi-local GGA functionals, namely, BEEF-vdW [53], PBE [34], and PBEsol [54], as well as the $\mathrm{DFT}(\mathrm{PBE})+U(4.5)$ method to 1 monolayer (ML) CO adsorbed on the fully-oxidized $\mathrm{CeO}_{2}(111)$ surface modeled by $(1 \times 1)$ surface unit cells $($ see the Supporting Information for further details). Moreover, we have also employed the nonlocal HSE06 hybrid functional [43]. Additionally, the D3 dispersion correction (DFT+D3) $[55,56]$ has been used in conjunction with the PBE, PBEsol, and HSE06 functionals, as well as with the PBE+U approach. The calculated $\mathrm{CO}$ adsorption energies $\lesssim 0.3 \mathrm{eV}$ (in absolute value) with the GGA and GGA $+U$ methods (Tables 1 and S3) are consistent with those of previous PBE/PW91 [28, 29], PBE/PW91+U(4.5/5) [27, 28], and $\mathrm{PBE}+U(4.5)+\mathrm{D} 3$ analyses. The HSE06 adsorption energy is similar to that calculated with $\mathrm{PBE}+U(4.5)$ within less than $0.1 \mathrm{eV}$ (Table 1). 
Independent of the employed DFT method, the most energetically preferable adsorption site on the $\mathrm{CeO}_{2}(111)$ surface is atop $\mathrm{Ce}^{4+}$ with the $\mathrm{C}-\mathrm{O}$ bond (in most cases) perpendicular to the surface. Interestingly, the distance of the $\mathrm{C}$ atom to the $\mathrm{Ce}^{4+}, d_{\mathrm{Ce}-\mathrm{C}}$, and the change of the $\mathrm{C}-\mathrm{O}$ bond length upon adsorption with respect to the calculated value for the gas phase molecule, $\Delta d_{\mathrm{C}-\mathrm{O}}$, depend on the approximation to the exchange-correlation functional in DFT (Tables 1 and S3). The $d_{\mathrm{Ce}-\mathrm{C}}$ distance is predicted to be shorter with most GGA-type functionals compared to that obtained with the hybrid HSE06 functional. Moreover, we found the $\mathrm{C}-\mathrm{O}$ bond lengthened by about $0.1 \mathrm{pm}$ for all GGA-type functionals, but shortened for the hybrid HSE06 functional by about the same amount (Tables 1 and S3).

The CO stretching vibrational frequency computed with the GGA/GGA $+U$ approximations (Tables 1 and S3) are by $6-10 \mathrm{~cm}^{-1}$ smaller than the experimental value of 2154 $\mathrm{cm}^{-1}$ (Fig. 1) and the calculated blueshifts of +1 to $+5 \mathrm{~cm}^{-1}$ are systematically smaller than the experimental value of $\Delta v=+11 \mathrm{~cm}^{-1}$. However, the hybrid HSE06 functional predicts values of $v=2157 \mathrm{~cm}^{-1}$ and $\Delta v=+14 \mathrm{~cm}^{-1}$ that are in good agreement with the experiment (Table 1).

On the (110) surface, a similar adsorption site for $\mathrm{CO}$ atop of a $\mathrm{Ce}^{4+}$ ion is found with the $\mathrm{C}-\mathrm{O}$ bond nearly perpendicular to the surface, as well as two strongly tilted configurations (Fig. 1). The difference between the tilted configurations is that in one case the $\mathrm{CO}$ molecule points to the $\mathrm{Ce}^{4+}$ ion in the second oxide plane (tilt.1, Fig. S1 c, g) and in the other one to the $\mathrm{Ce}^{4+}$ ion in the surface along the [1-10] direction (tilt.2, Fig. S1d, h). In the literature [27-29], in addition to the atop $\mathrm{Ce}^{4+}$ site, the formation of strongly bound carbonate $\mathrm{CO}_{3}{ }^{2-}$-like species and the reduction of the ceria surface have been reported, but such species have not been observed in the IRRAS experiment [15]. The $\mathrm{PBE}+U(4.5)$ calculated binding energy for the atop $\mathrm{Ce}^{4+}$ site $\lesssim 0.2 \mathrm{eV}$ (in absolute value, Table 1) is consistent with that of previous PBE/PW91+U(4.5/5) $[27,28]$ and PBE/PW91 $[28,29]$ studies. In the two tilted configurations, the adsorption energy is practically the same and is by less than $0.1 \mathrm{eV}$ larger than in the atop one (Table 1). Once again, the HSE06 calculated binding energies are comparable to those obtained with $\mathrm{PBE}+U(4.5)$.

As mentioned above, for $\mathrm{CO}$ adsorption on the $\mathrm{CeO}_{2}(110)$ surface two blue shifted peaks by $\Delta v=+28$ and $+17 \mathrm{~cm}^{-1}$ are observed (Fig. 1). Inspection of Table 1 reveals that similar to the case of the (111) surface, for the (110), the deviations of the computed $v$ and $\Delta v$ values with $\mathrm{PBE}+U(4.5)$ from the experimental ones are substantial and that only the HSE06 values are in good agreement with the results of the IRRAS experiment with deviations $\lesssim 9 \mathrm{~cm}^{-1}$. The more intense band at $2171 \mathrm{~cm}^{-1}$ and the less intense one at $2160 \mathrm{~cm}^{-1}$ are here assigned to CO bound to $\mathrm{Ce}^{4+}$ in atop and tilted configurations, respectively. 
Inspection of the structures for the adsorption of $\mathrm{CO}$ on the $\mathrm{CeO}_{2}(110)$ surfaces reveals that the $\mathrm{Ce}-\mathrm{C}$ distances are shorter with $\mathrm{PBE}+U$ than with HSE06 (Table 1, Fig. S1), which is similar to the case of the $\mathrm{CeO}_{2}(111)$ surface. Also, the $\mathrm{C}-\mathrm{O}$ bond length is longer with $\mathrm{PBE}+U$ than with HSE06, and the corresponding changes in the $\mathrm{C}-\mathrm{O}$ bond length, $\Delta d_{\mathrm{C}-\mathrm{O}}$, upon adsorption show that (in most cases) $\Delta d_{\mathrm{C}-\mathrm{O}}>0$ with $\mathrm{PBE}+U$ and $\Delta d_{\mathrm{C}-\mathrm{O}}<0$ with HSE06 (Table 1). Specifically, the $\mathrm{C}-\mathrm{O}$ bond is calculated to be compressed by $0.18 \mathrm{pm}$ and up to about $0.1 \mathrm{pm}$ in the atop and tilted configurations, respectively, by the HSE06 functional. However, for the tilted configurations, the $\mathrm{PBE}+U(4.5)$ calculated $\mathrm{C}-\mathrm{O}$ bonds are stretched by about $0.1 \mathrm{pm}$. A compression of about $0.1 \mathrm{pm}$ has been previously calculated for the atop $\mathrm{Ce}^{4+}$ configuration employing the hybrid B3LYP functional [30,31].

In summary, with the $\operatorname{PBE}+U$ functional the agreement of theoretical vibrational frequencies with the IRRAS experiment is rather poor, while with the HSE06 approach almost quantitative agreement can be achieved. To shed more light on this problem, we further inspected the density of states (DOS) for the $\mathrm{CO}$ adsorbed systems compared to that for the $\mathrm{CO}$ molecule in the gas phase. As an example, we focus here on the atop Ce configuration on the (111) surface (Fig. 3 and Fig. S4). The electronic state reminiscent of the $\mathrm{CO} 5 \sigma$ orbital is clearly perturbed upon adsorption (Fig. 2a, b). The shorter $\mathrm{Ce}-\mathrm{C}$ distance obtained with $\mathrm{PBE}+U$ compared to HSE06 relates to a stronger $\sigma$-donation type of interaction by which charge is donated to the empty Ce $d$-states, as evidenced by the more pronounced electron density accumulation at the $\mathrm{Ce}-\mathrm{C}$ bond (cf. Figs. 2c, d). As a consequence of the $\sigma$-donation, a shortening of the $\mathrm{C}-\mathrm{O}$ bond is expected due to bond depopulation which implies $\Delta d_{\mathrm{C}-\mathrm{O}}<0$. However, as stated above, it is only with the HSE06 functional that the computed $\Delta d_{\mathrm{C}-\mathrm{O}}$ values are negative, and those obtained with $\mathrm{PBE}+U$ are positive.

PBE+U
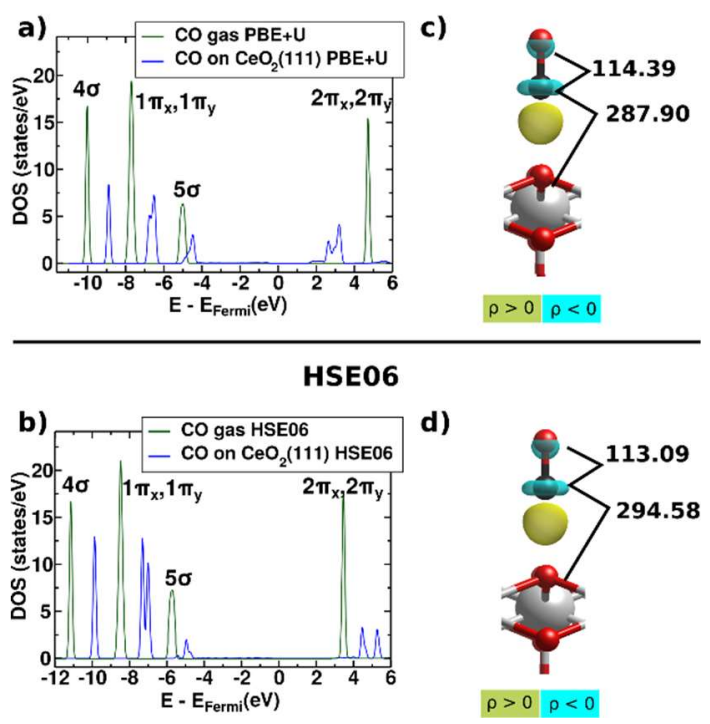
Figure 2. Total density of states (DOS) projected onto the $\mathrm{CO}$ (blue) for $1 \mathrm{ML} \mathrm{CO}$ adsorbed on the $\mathrm{CeO}_{2}(111)$ surface in the atop $\mathrm{Ce}^{4+}$ configuration with the (a) $\mathrm{PBE}+U(4.5)$ and (c) HSE06 approaches compared to that of $\mathrm{CO}$ in the gas phase. (b) and (d) Isosurfaces of the charge density difference, namely, that of the $\mathrm{CO}$ adsorbed system from which both the charge density of the clean $\mathrm{CeO}_{2}(111)$ surface (with a structure corresponding to that of the adsorbed system) and that of the layer of adsorbed $\mathrm{CO}$ molecules have been subtracted. Selected interatomic distances (in pm) are indicated.

In addition, the $\mathrm{PBE}+U$ derived charge density difference upon $\mathrm{CO}$ adsorption on the $\mathrm{CeO}_{2}$ (111) surface (Figs. 2c) reveals that electron density on the $\mathrm{O}$ atom of the molecule is enhanced, indicating that antibonding orbitals of $\mathrm{CO}$ have been populated. In the HSE06 calculations this electron density enhancement on the $\mathrm{O}$ end is less noticeable (Fig. 2d). As a result of the surface $\rightarrow \mathrm{CO}$ backdonation type of interaction, a lengthening of the $\mathrm{C}-\mathrm{O}$ bond is expected due to bond population. The overall change in the $\mathrm{C}-\mathrm{O}$ bond upon adsorption is the result of synergistic charge transfer effects, namely, $\mathrm{CO} \rightarrow$ surface donation and surface $\rightarrow \mathrm{CO}$ backdonation, which cause the shortening and lengthening of the $\mathrm{C}-\mathrm{O}$ bond length respectively. The $\mathrm{PBE}+U$ approach predicts larger $\mathrm{CO} \rightarrow$ surface and surface $\rightarrow \mathrm{CO}$ charge transfers compared to HSE06 and as a result of which $\Delta d_{\mathrm{C}-\mathrm{O}}>0$. Similar findings were obtained for $1 \mathrm{ML}$ CO adsorption on the $\mathrm{CeO}_{2}(110)$ surface (Figs. S5 and S6). The $0.25 \mathrm{ML} \mathrm{CO}$ adsorption on both surfaces has also been considered (see Figs. S2 and S3).

The calculated $\mathrm{CO}$ vibrational frequency of the gas phase molecule as a function of a varying $\mathrm{C}-\mathrm{O}$ bond length shows a clear inverse correlation (Fig. S7). The shorter the $\mathrm{C}-\mathrm{O}$ bond, the higher the vibrational CO stretching frequency leading to a blueshift, whereas the longer the $\mathrm{C}-\mathrm{O}$ bond, the lower the frequency, resulting in a redshift. The HSE06 computed $\Delta v$ and $\Delta d_{\mathrm{C}-\mathrm{O}}$ changes upon $\mathrm{CO}$ adsorption on the $\mathrm{CeO}_{2}(111)$ and (110) surfaces, qualitatively follow the inverse correlation. However, with $\mathrm{PBE}+U$ deviations are significant.

Having established the ability of the HSE06 functional to provide reliable $\mathrm{CO}$ vibrational frequency shifts of $\mathrm{CO}$ adsorbed on ceria surfaces upon comparison with the experimental IRRAS data (selected calculations using the PBE0 [57] functional give similar results, see Supporting Information), we note the important result that the shifts are facet- and configuration-dependent, because the final changes in the $\mathrm{C}-\mathrm{O}$ bond length upon adsorption depend on them too, and thus $\mathrm{CO}$ can be used as a sensitive chemical probe molecule in the characterization of the specific facets exposed by ceria catalysts. Specifically, for the atop configurations on the (110) and (111) surfaces, blueshifts of the CO stretching frequency of +21 and $+14 \mathrm{~cm}^{-1}$, respectively, have been computed and related to a shortening of the $\mathrm{C}-\mathrm{O}$ bond of 0.18 and $0.06 \mathrm{pm}$, respectively. Moreover, an additional blueshifted frequency of $+7 \mathrm{~cm}^{-1}$ is calculated for the (110) surface and related to the shortening of $0.07 \mathrm{pm}$ of the $\mathrm{C}-\mathrm{O}$ bond of a tilted adsorbed $\mathrm{CO}$. Facet-specific donation and backdonation effects were revealed no only on $\mathrm{CeO}_{2}$, as demonstrated in this work, but also on $\mathrm{TiO}_{2}$ anatase nanoparticles exposing the $\{101\}$ and $\{110\}$ facets [58]. 
Generally speaking, the characterization of metal oxide nanoparticles under realistic conditions is of great importance for catalytic applications and is paramount for exploiting the interplay between structure and reactivity. A rather large variety of different vibrational frequencies can be observed for powder catalysts when using IR probe molecules, and a solid basis for a thorough assignment is needed. DFT calculations validated by IRRAS data for welldefined single crystals can provide such a reliable reference. The validation process here described, demonstrated a failure of the frequently used GGA functionals in DFT, for the example of $\mathrm{CeO}_{2}$-bound $\mathrm{CO}$. As a consequence, earlier interpretations of measured spectra based on GGA calculations may need to be revised. Importantly, the HSE06 approach provides the required accuracy and allows reproducing the facet- and configuration-dependent vibrational spectra for $\mathrm{CO}$ adsorbed on the $\mathrm{CeO}_{2}(111)$ and (110) surfaces. This high accuracy achieved for one of the most challenging oxide surface manifests a striking advancement in the theoretical description of metal-oxides and opens up the path for analyzing more complex scenarios in future work, including oxygen vacancies and metal adatoms.

\section{Acknowledgements}

This project received funding from the European Union's Horizon 2020 research and innovation programme under the Marie Skłodowska-Curie grant agreement No 832121. Computer time provided by the BIFI-ZCAM and the RES (Red Española de Supercomputación) resources at MareNostrum 4 (BSC, Barcelona) and Altamira (IFCA, Cantabria) nodes, as well as by the DECI resources at Finis Terrae II based in Spain at CESGA, with the support from PRACE aislb, is acknowledge. M.V.G.P. thanks the support by the MICINN-Spain (RTI2018-101604-BI00). The DFT data that support the findings of this study are available in Materials Cloud $\{$ https://www.materialscloud.org/home\} with the identifier doi: 10.24435/materialscloud:z7-z0.

\section{References}

[1] Catalysis by Ceria and Related Materials, edited by A. Trovarelli and P. Fornasiero, 2nd ed. (Imperial College Press, London, United Kingdom, 2013).

[2] S. Park, J. M. Vohs, and R. J. Gorte, Direct Oxidation of Hydrocarbons in a Solid-Oxide Fuel Cell, Nature 404, 265 (2000).

[3] C. Xu and X. Qu, Cerium Oxide Nanoparticle: A Remarkably Versatile Rare Earth Nanomaterial for Biological Applications, NPG Asia Mater. 6, e90 (2014).

[4] G. Pulido-Reyes, I. Rodea-Palomares, S. Das, T. S. Sakthivel, F. Leganes, R. Rosal, S. Seal, and F. Fernández-Piñas, Untangling the Biological Effects of Cerium Oxide Nanoparticles: The Role of Surface Valence States, Sci. Rep. 5, 15613 (2015).

[5] D. Mullins, P. Albrecht, and F. Calaza, Variations in Reactivity on Different Crystallographic Orientations of Cerium Oxide, Top. Catal. 56, 1345 (2013). 
[6] M. Melchionna and P. Fornasiero, The Role of Ceria-Based Nanostructured Materials in Energy Applications, Mater. Today 17, 349 (2014).

[7] W. Huang and Y. Gao, Morphology-Dependent Surface Chemistry and Catalysis of $\mathrm{CeO}_{2} \mathrm{Nanocrystals}_{\text {, }}$ Catal. Sci. Technol. 4, 3772 (2014).

[8] A. Trovarelli and J. Llorca, Ceria Catalysts at Nanoscale: How Do Crystal Shapes Shape Catalysis? ACS Catal. 7, 4716 (2017).

[9] Z. Hu, X. Liu, D. Meng, Y. Guo, Y. Guo, and G. Lu, Effect of Ceria Crystal Plane on the Physicochemical and Catalytic Properties of Pd/Ceria for CO and Propane Oxidation, ACS Catal. 6, 2265 (2016).

[10] G. Vilé, S. Colussi, F. Krumeich, A. Trovarelli, and J. Pérez-Ramírez, Opposite Face Sensitivity of $\mathrm{CeO}_{2}$ in Hydrogenation and Oxidation Catalysis, Angew. Chem. Int. Ed. 53, 12069 (2014).

[11] Y. Cao, L. Zhao, T. Gutmann, Y. Xu, L. Dong, G. Buntkowsky, and F. Gao, Getting Insights into the Influence of Crystal Plane Effect of Shaped Ceria on Its Catalytic Performances, J. Phys. Chem. C 122, 20402 (2018).

[12] C. Li, Y. Sun, I. Djerdj, P. Voepel, C.-C. Sack, T. Weller, R. Ellinghaus, J. Sann, Y. Guo, B. M. Smarsly, and H. Over, Shape-Controlled $\mathrm{CeO}_{2}$ Nanoparticles: Stability and Activity in the Catalyzed $\mathrm{HCl}$ Oxidation Reaction, ACS Catal. 7, 6453 (2017).

[13] I. Florea, C. Feral-Martin, J. Majimel, D. Ihiawakrim, C.Hirlimann, and O. Ersen, Three-Dimensional Tomographic Analyses of $\mathrm{CeO}_{2}$ Nanoparticles, Cryst. Growth Des. 13, 1110 (2013).

[14] J. Chen, X. Wu, M. A. Hope, K. Qian, D. M. Halat, T. Liu, Y. Li, L. Shen, X. Ke, Y. Wen et al., Polar Surface Structure of Oxide Nanocrystals Revealed with Solid-State NMR Spectroscopy, Nat. Commun. 10, 5420 (2019).

[15] C. Yang, X. Yu, S. Heißler, A. Nefedov, S. Colussi, J. Llorca, A. Trovarelli, Y. Wang, and C, Wöll, Surface Faceting and Reconstruction of Ceria Nanoparticles, Angew. Chem. Int. Ed. 56, 375 (2017).

[16] P. A. Crozier, R. Wang, and R. Sharma, In Situ Environmental TEM Studies of Dynamic Changes in Cerium-Based Oxides Nanoparticles During Redox Processes, Ultramicroscopy 108, 1432 (2008).

[17] O. S. Bezkrovnyi, P. Kraszkiewicz, M. Ptak, and L. Kepinski, Thermally Induced Reconstruction of Ceria Nanocubes into Zigzag \{111\}-Nanofacetted Structures and Its Influence on Catalytic Activity in CO Oxidation, Catal. Commun. 117, 94 (2018).

[18] A. M. Abdel-Mageed, A. Klyushin, A. Rezvani, A. Knop-Gericke, R. Schlögl, and J. Behm, Negative Charging of $\mathrm{Au}$ Nanoparticles during Methanol Synthesis from $\mathrm{CO}_{2} / \mathrm{H}_{2}$ on a $\mathrm{Au} / \mathrm{ZnO}$ Catalyst: Insights from Operando IR and Near-Ambient-Pressure XPS and XAS Measurements, Angew. Chem. Int. Ed. 58, 10325 (2019).

[19] M. I. Zaki, B. Vielhaber, and H. Knoezinger, Low-Temperature Carbon Monoxide Adsorption and State of Molybdena Supported on Alumina, Titania, Ceria, and Zirconia. An Infrared Spectroscopic investigation, J. Phys. Chem. 90, 3176 (1986).

[20] C. Li, Y. Sakata, T. Arai, K. Domen, K.-i. Maruya, and T. Onishi, Carbon Monoxide and Carbon Dioxide Adsorption on Cerium Oxide Studied by Fourier-Transform Infrared Spectroscopy. Part 1.Formation of Carbonate Species on Dehydroxylated $\mathrm{CeO}_{2}$, at Room Temperature, J. Chem. Soc., Faraday Trans. 1 85, 929 (1989).

[21] C. Binet, M. Daturi, and J.-C. Lavalley, IR Study of Polycrystalline Ceria Properties in Oxidised and Reduced States, Catal. Today 50, 207 (1999). 
[22] R. Farra, S. Wrabetz, M. E. Schuster, E. Stotz, N. G. Hamilton, A. P. Amrute, J. Pérez-Ramírez, N. López, and D. Teschner, Understanding $\mathrm{CeO}_{2}$ as a Deacon Catalyst by Probe Molecule Adsorption and In Situ Infrared Characterisations, Phys. Chem. Chem. Phys. 15, 3454 (2013).

[23] S. Agarwal, X. Zhu, E. J. M. Hensen, B. L. Mojet, and L. Lefferts, Surface-Dependence of Defect Chemistry of Nanostructured Ceria, J. Phys. Chem. C 119, 12423 (2015).

[24] C. Wöll, Structure and Chemical Properties of Oxide Nanoparticles Determined by Surface-Ligand IR Spectroscopy, ACS Catal. 10, 168 (2020).

[25] C. Yang, L.-L. Yin, F. Bebensee, M. Buchholz, H. Sezen, S. Heissler, J. Chen, A. Nefedov, H. Idriss, X.-Q. Gong, and C. Wöll, Chemical Activity of Oxygen Vacancies on Ceria: A Combined Experimental and Theoretical Study on $\mathrm{CeO}_{2}(111)$, Phys. Chem. Chem. Phys. 16, 24165 (2014).

[26] C. Yang, X. Yu, S. Heißler, P. G. Weidler, A. Nefedov, Y. Wang, C. Wöll, T. Kropp, J. Paier, and J. Sauer, $\mathrm{O}_{2}$ Activation on Ceria Catalysts -The Importance of Substrate Crystallographic Orientation, Angew. Chem. Int. Ed. 56, 16399 (2017).

[27] M. Nolan and G. W. Watson, The Surface Dependence of CO Adsorption on Ceria, J. Phys. Chem. B 110, 16600 (2006).

[28] M. Huang and S. Fabris, CO Adsorption and Oxidation on Ceria Surfaces from DFT+U Calculations, J. Phys. Chem. C 112, 23, 8643 (2008).

[29] Z. Yang, T. K. Woo, and K. Hermansson, Strong and Weak Adsorption of CO on $\mathrm{CeO}_{2}$ Surfaces from First Principles Calculations, Chem. Phys. Lett. 396, 384 (2004).

[30] C. Müller, C. Freysoldt, M. Baudin, and K. Hermansson, An Ab Initio Study of CO Adsorption on Ceria(110), Chem. Phys. 318, 180 (2005).

[31] C. Müller, B. Herschend, K. Hermansson, and B. Paulus, Application of the Method of Increments to the Adsorption of $\mathrm{CO}$ on the $\mathrm{CeO}_{2}$ (110) Surface, J. Chem. Phys. 128, 214701 (2008).

[32] G. Pacchioni, G. Cogliandro, and P. S. Bagus, Characterization of Oxide Surfaces by Infrared Spectroscopy of Adsorbed Carbon Monoxide: A Theoretical Investigation of the Frequency Shift of CO on $\mathrm{MgO}$ and NiO, Surf. Sci. 255, 344 (1991).

[33] S. L. Dudarev, G. A. Botton, S. Y. Savrasov, C. J. Humphreys, A. P. Sutton, Electron-Energy-Loss Spectra and the Structural Stability of Nickel Oxide: An LSDA+U Study, Phys. Rev. B 57, 1505 (1998).

[34] J. P. Perdew, K. Burke, and M. Ernzerhof, Generalized Gradient Approximation Made Simple, Phys. Rev. Lett. 77, 3865 (1996).

[35] S. Fabris, G. Vicario, G. Balducci, S. de Gironcoli, and S. Baroni, Electronic and Atomistic Structures of Clean and Reduced Ceria Surfaces, J. Phys. Chem. B 109, 22860 (2005).

[36] G. Kresse, P. Blaha, J. L. F. da Silva, and M. V. Ganduglia-Pirovano, Comment on: Taming Multiple Valency with Density Functionals: A Case Study of Defective Ceria, Phys. Rev. B 72, 237101 (2005).

[37] M. V. Ganduglia-Pirovano, A. Hofmann, and J. Sauer, Oxygen Vacancies in Transition Metal and Rare Earth Oxides: Current State of Understanding and Remaining Challenges, Surf. Sci. Rep. 62, 219 (2007).

[38] J. L. F. Da Silva, M. V. Ganduglia-Pirovano, J. Sauer, V. Bayer, and G. Kresse, Hybrid Functionals Applied to Rare-Earth Oxides: The Example of Ceria, Phys. Rev. B 75, 045121 (2007). 
[39] C. W. M. Castleton, J. Kullgren, K. Hermansson, Tuning LDA+U for Electron Localization and Structure at Oxygen Vacancies in Ceria, J. Chem. Phys. 127, 244704 (2007)

[40] D. A. Andersson, S. I. Simak, B. Johansson, I. A. Abrikosov, and N. V. Skorodumova, Modeling of $\mathrm{CeO}_{2}, \mathrm{Ce}_{2} \mathrm{O}_{3}$, and $\mathrm{CeO}_{2-\mathrm{x}}$ in the LDA+U Formalism. Phys. Rev. B75, 035109 (2007).

[41] C. Loschen, J. Carrasco, K. M. Neyman, and F. Illas, F. First-Principles LDA+U and GGA+U Study of Cerium Oxides: Dependence on the Effective U Parameter, Phys. Rev. B 75, 035115 (2007).

[42] D. Du, M. J. Wolf, K. Hermansson, and P. Broqvist, Screened Hybrid Functionals Applied to Ceria: Effect of Fock Exchange, Phys. Rev. B 97, 235203 (2018).

[43] A.V. Krukau, O. A. Vydrov, A. F. Izmaylov, and G. E. Scuseria, Influence of the Exchange Screening Parameter on the Performance of Screened Hybrid Functionals, J. Chem. Phys. 125, 224106 (2006).

[44] M. V. Ganduglia-Pirovano, J. L. F. Da Silva, and J. Sauer, Density functional calculations of the structure of near-surface oxygen vacancies and electron localization on $\mathrm{CeO}_{2}(111)$, Phys. Rev. Lett. 102, $026101(2009)$

[45] J. Paier, C. Penschke, and J. Sauer, Oxygen Defects and Surface Chemistry of Ceria: Quantum Chemical Studies Compared to Experiment, Chem. Rev. 113, 6, 3949 (2013).

[46] J. Graciani, A. M. Márquez, J. J. Plata, Y. Ortega, N. C. Hernández, A. Meyer, C. M. Zicovich-Wilson, and J. Fdez. Sanz, Comparative Study on the Performance of Hybrid DFT Functionals in Highly Correlated Oxides: The Case of $\mathrm{CeO}_{2}$ and $\mathrm{Ce}_{2} \mathrm{O}_{3}$, J. Chem. Theory Comput. 7, 56 (2011).

[47] Kh. E. El-Kelany, C. Ravoux, J. K. Desmarais, P. Cortona, Y. Pan, J. S. Tse, and A. Erba, Spin Localization, Magnetic Ordering, and Electronic Properties of Strongly Correlated $\mathrm{Ln}_{2} \mathrm{O}_{3}$ Sesquioxides (Ln=La, Ce, Pr, Nd), Phys. Rev. B 97, 245118 (2018).

[48] B. Civalleri., L. Maschio, P. Ugliengo, and C. M. Zicovich-Wilson, Role of Dispersive Interactions in the $\mathrm{CO}$ Adsorption on $\mathrm{MgO}(001)$ : Periodic B3LYP Calculations Augmented with an Empirical Dispersion Term, Phys. Chem. Chem. Phys. 12, 6382 (2010).

[49] J. Maul, G. Spoto, L. Mino, and A. Erba, Elucidating the Structure and Dynamics of CO Ad-layers on MgO Surfaces, Phys. Chem. Chem. Phys. 21, 26279-26283 (2019).

[50] Y. Wang, S. de Gironcoli, N. S. Hush, and J. R. Reimers, Successful a Priori Modeling of CO Adsorption on Pt(111) Using Periodic Hybrid Density Functional Theory, J. Am. Chem. Soc. 129, 10403 (2007).

[51] G. A. H. Mekhemer and M. I. Zaki, Low-Temperature IR Spectroscopy of CO Adsorption on Calcined Supported $\mathrm{CeO}_{2}$ : Probing Adsorbed Species and Adsorbing Sites, Adsorpt. Sci. Technol. 15, 377 (1997).

[52] Y. Wang and Ch. Wöll, IR Spectroscopic Investigations of Chemical and Photochemical Reactions on Metal Oxides: Bridging the Materials Gap, Chem. Soc. Rev. 46, 1875 (2017).

[53] J. Wellendorff, K. T. Lundgaard, A. Møgelhøj, V. Petzold, D. D. Landis, J. K. Nørskov, T. Bligaard, and K. W. Jacobsen, Density Functionals for Surface Science: Exchange-Correlation Model Development with Bayesian Error Estimation, Phys. Rev. B 85, 235149 (2012).

[54] G. I. Csonka, J. P. Perdew, A. Ruzsinszky, P. H. T. Philipsen, S. Lebègue, J. Paier, O. A. Vydrov, and J. G. Ángyán, Assessing the Performance of Recent Density Functionals for Bulk Solids, Phys. Rev. B 79, 155107 (2009). 
[55] S. Grimme, J. Antony, S. Ehrlich, and S. Krieg, A Consistent and Accurate Ab Initio Parametrization of Density Functional Dispersion Correction (DFT-D) for the 94 Elements H-Pu, J. Chem. Phys. 132, 154104 (2010).

[56] S. Grimme, S. Ehrlich, and L. Goerigk, Effect of the Damping Function in Dispersion Corrected Density Functional Theory, J. Comput. Chem. 32, 1456 (2011).

[57] J. P. Perdew and M. Ernzerhof, Rationale for Mixing Exact Exchange with Density Functional Approximations, J. Chem. Phys. 105, 9982 (1996).

[58] C. Deiana, E. Fois, G. Martra, S. Narbey, F.Pellegrino, and G. Tabacchi, On the Simple Complexity of Carbon Monoxide on Oxide Surfaces: Facet-Specific Donation and Backdonation Effects Revealed on $\mathrm{TiO}_{2}$ Anatase Nanoparticles, Chem. Phys. Chem. 17, 1956 (2016).

[59] See Supplemental Material [url] for the description of the methods and models used in this work, which includes Refs. [60-69].

[60] G. Kresse and J. Furthmuller, Efficient Iterative Schemes for ab initio Total-energy Calculations Using a Plane-Wave Basis Set, Phys. Rev. B 5411169 (1996).

[61] G. Kresse and J. Furthmuller, Efficiency of Ab-initio Total Energy Calculations for Metals and Semiconductors Using a Plane-Wave Basis Set, Comput. Mater. Sci. 6 15-50 (1996).

[62] S.R. Bahn and K.W. Jacobsen, An Object-oriented Scripting Interface to a Legacy Electronic Structure Code, Comput. Sci. Eng. 4 56-66 (2002).

[63] P.E. Blöchl, Projector Augmented-wave Method, Phys. Rev. B 5017953 (1994).

[64] G. Kresse and J. Joubert, From Ultrasoft Pseudopotentials to the Projector Augmented-wave Method, Phys. Rev. B 591758 (1999).

[65] J. Wellendorff, K.T. Lundgaard, A. Møgelhøj, V. Petzold, D.D. Landis, J.K. Nørskov, T. Bligaard, and K.W. Jacobsen, Density Functionals for Surface Science: Exchange-correlation Model Development with Bayesian Error Estimation, Phys. Rev. B 85235149 (2012).

[66] P. Perdew, A. Ruzsinszky, G. I. Csonka, O. A. Vydrov, G. E. Scuseria, L. A. Constrantin, X. Zhou, and K. Burke, Restoring the Density-Gradient Expansion for Exchange in Solids and Surfaces, Phys. Rev. Lett. 100136406 (2008).

[67] A.V. Krukau, O. A. Vydrov, A. F. Izmaylov, and G. E. Scuseria, Influence of the Exchange Screening Parameter on the Performance of Screened Hybrid Functionals, J. Chem. Phys. 125224106 (2006).

[68] H.J. Monkhorst and J.D. Pack, Special Points for Brillouin-zone Integrations, Phys. Rev. B 135188 (1976).

[69] G. A. H. Mekhemer and M. I. Zaki, Low-Temperature IR Spectroscopy of CO Adsorption on Calcined Supported $\mathrm{CeO}_{2}$ : Probing Adsorbed Species and Adsorbing Sites, Adsorp. Sci. Technol. 15 377-389 (1997). 